\title{
Realizing Expressions of Doubt in Collaborative Dialogue *
}

\author{
Leah Schroeder and Sandra Carberry \\ Computer and Information Sciences \\ University of Delaware \\ Newark, DE 19716 \\ $\{$ schroede, carberry $\} @$ cis.udel.edu
}

\begin{abstract}
One way to begin a negotiation subdialogue is to express doubt at a proposition. However, expressions of doubt occur in a variety of forms, each of which conveys information about the nature of the doubt that is important for the subsequent resolution of the conflict. This paper presents our work on realizing expressions of doubt appropriately in natural language dialogues.
\end{abstract}

\section{Introduction}

Participants in a collaborative natural language dialogue must develop mutual beliefs about what is said, what is meant, and the implications for the task at hand. We may think of each utterance as a proposed change to the agents' common ground (Clark, 1996). Sincc autonomous agents enter the dialogue with differing domain, world, and personal knowledge, it is inevitable that some beliefs conveyed by an utterance will not be accepted because they conflict with existing beliefs of the agent. However, it is also the case that these conflicting beliefs will not, necessarily result in rejection of the proposed beliefs, but in subdialogues to negotiate a modification that, is acceptable to both agents(Chu-Carroll and Carberry, 1995). One way to begin such a subdialogue is to express doubt at the beliefs proposed by an utterance. In the following example, the boldface utterance is expressing doubt at the previous utterance $^{1}$ (Transcripts, 1982) ${ }^{2}$ :

$\mathrm{H}$ : and - there's no reason why you shouldn't have an ira for last $y \mathrm{r}$

$\mathrm{J}$ : well $\mathrm{i}$ thought they just started this $\mathrm{yr}$

\footnotetext{
* This work was supported by NSF grant \#GElR-9354869 and \#CDA-9703088

1 Throughout this paper I use the phrase "doubt at an utterance" in place of "doubt at a proposition conveyed or implied by an utterance." I do not mean the utterance itself is somchow doubted, but that the utterance introduced the object of doubt into the dialogue. It may be the case that the agent is doubting a proposition expressed in the utterance, or doubting the optimality of, or ability to execute, an action suggested in the utterance.

${ }^{2}$ All of the examples in this paper, except where otherwise noted, are from this source.
}

$\mathrm{H}$ : oh no. ira's were available as long as you are not a participant in an existing pension

An expression of doubt is an utterance that conveys uncertain disbelief in a proposition that was introduced in an earlicr utterance. An expression of doubt signals that the speaker does not accept the utterance at which she is expressing doubt, but she is neither expressing a "ncutral" attitude toward it nor rejecting it with certainty ${ }^{3}$. In the above example, J cannot be said to be rejecting the proposal outright, because her response indicates that she is uncertain in her disbelief.

A natural language system must be able to express doubt, particularly in cases where it has incomplete or uncertain knowledge. Examination of natural language corpora shows that expressions of doubt may be realized in a varicty of forms. Furthermore, the form of the utterance conveys information about the nature of the doubt that is important for the subsequent resolution of the conflict. Thus a collaborative natural language system must be able to generate utterances that convey doubt naturally and effectively. This paper presents our work on realizing expressions of doubt appropriately in natural language dialogues.

\section{Previous Work}

In Chu-Carroll and Carberry (1998) the collaborative planning process is modeled as a ProposeEvaluate-Modify cycle, in which an agent is able to detect conflicts in belief and initiate collaborative negotiation subdialogues to attempt to resolve the conflicts. They use a modified version of Galliers belief revision mechanism(Galliers, 1992; Logan et al., 1994) to determine whether to accept a proposition and in determining which conflicting beliefs to use to refute an utterance that is not accepted. However, their work does not address how an expression of doubt should be realized in a natural language utterance.

Vander Linden and Di Eugenio (Vander Linden and Di Eugenio, 1996) studied negative imperatives

\footnotetext{
${ }^{3}$ Absolute rejection may be expressed as doubt for the sake of politeness. We do not address that issue here.
} 
in instructional texts. They used machine learning to correlate features of an action $X^{\prime}$ s relationship to the reader in terms of attention, awareness, and safety, with whether it was realized as Don't $X$, Never $X$, or Take care not to $X$. In our research, we draw on their notion of identifying how features of the generation context correlate with how an utterance should be expressed. However, our work differs from theirs in that we must deal with an agent's belicfs motivating his doubt and we consider a wider range of variations in realization.

\section{Communicating an Expression of Doubt}

We assume appropriate mechanisms for detecting conflict and determining when to engage in a subdialogue by expressing doubt(Chu-Carroll and Carbetry, 1998), as well as an appropriate belicf revision mechanism, and in this papor concentrate on how an expression of doubt should be realized as an utterance. A cooperative agent should be as informative as needed, without expressing too much irrelevant information(Grice, 1975). Thus, in formulating an expression of doubt, we must consider how much the doubted agent needs to know in order to collaborate in resolving the doubt and how much we can expect him to infer without being explicitly told. In addition, Clark (1996) argues that participants in discourse select utterances that express their communicative intent efficiently, often in sentence fragments. Since such efficiency of expression is the expected natural form of discourse, a hearer is likely to dorive unintended implications from significantly less economical realizations.

Expressions of doubt, by our definition, signal nonacceptance because of 'uncertain disbelief. In order for the doubted agent to attempt to collaborate in resolving the doubt, he needs to know several things. Most basically, he needs to recognize that there is doubt in a particular utterance. In the absence of an objection to an utterance, the speaker will assume an implicit acceptance(Lambert and Carborry, 1999). To efficiently negotiate an acceptable resolution to the bolicf conflict, idcally the doubted agent must know something about the beliefs of the doubting agent; in particular, which belicf(s) are causing her nonacceptance, and the stuength of these beliefs. If the doubted agent decides to retain his original beliefs, this information helps him to construct an argument that will be maximally effective and efficient in his attempt to convince the doubting agent(Chu-Carroll and Carberry, 1998).

To identify how expressions of doubt are realized in naturally occurring dialogue and how these roalizations convey the requisite belicfs, we analyzed features of individual expressions of doubt extracted from natural corpora, and corrclated the various forms of the utterances with the features of the underlying beliefs. However, as explained in Section 3.3 , the use of machine learning techniques was not appropriate due to the nature of our corpus. Section 3.1 discusses features of underlying beliefs and Section 3.2 discusses the various forms that an $\mathrm{cx}-$ pression of doubt can take. Section 3.3 then presents a set of rules that relate the two.

\subsection{Belief features}

As noted above, beliefs play a prominent role in expressions of doubt, since a speaker will idcally convey enough information for the hearer to discern 1) that she is expressing doubt, 2) what she is doubting, 3) any support she has for the doubt, and 4) the strength of this support. In addition, spoakers tend to differentiate now supporting information from that, which is already part of the common ground and which should already have becn considered. These beliefs are often not explicitly stated, but are assumed to be inferable by the doubted agent, based on his knowledge of the previous dialogue, knowledge of the other agent's beliefs, a model of storcotypical beliefs, linguistic knowledge, and the particular realization of the doubting agent's utteraulece.

For example, consider the following assertion and two possible responses, each expressing doubt at, the proposition $P_{\text {doub }}$ that John Smith gets $\$ 800$ a month from Social Security ${ }^{4}$ :

\section{S: John Smith gets $\$ 800$ a month in Social Security.}

1) U: Isn't he less than 60 years old?

2) U: $\$ 800 \%$

In 1) U rolies on mutual domain knowledge to express doubt at $P_{\text {doubt }}$ by contending some other proposition $P_{i}$ that implies $\rightarrow P_{\text {doubt }}$ (Lambert and Carberry, 1999), namoly that Smith is younger than 62. In the rest of this paper, $P_{\text {doub }}$ refers to the doubted proposition and $P_{i}$ to a proposition other than $P_{\text {doubt }}$, if any, that is the reason for this doubt.

In addition, expectations also play a role in expressions of doubt. In the simplest case, the violated expcctation is just that $P_{\text {loubt }}$ is false. In other situations, an agent may have an expectation that a proposition will be false if instantiated with some particular subset of its possible instantiations. Responses that conflict with these expectations may provoke an expression of doubt, evon though the doubting agent may have little or no support for the expectation. Such violated expectations are often signaled by elliptical fragments, such as response 2) above where U conveys not only that she doesn't;

\footnotetext{
4'Jhis is not a naturally occurring example, but was made up for explanatory purposes.
} 
accept $P_{\text {doubt }}$, but also that her doubt stems from the instantiation of the amount term as $\$ 800$. We hypothesize that $U$ might accept a proposition with a different instantiation of the amount term, but $U$ doesn't explicitly state this, and other instantiations may be irrelevant. A violated expectation will be referred to as $P_{e}$ and is described further in Section 3.1.2. When and how these expectations arise is a topic for future research.

We assume the propositions $P_{\text {doubt }}, P_{c}$, and $P_{i}$, as well as the fact that we want to express doubt, as inputs to our generation process. Note that every expression of doubt will be associated with some $P_{\text {doubt }}$ and some $P_{e}$, since for every expression of doubt, there must be some doubted proposition and some inconsistency between the doubting agent's expectations and belicf in $P_{\text {doubt }}$. There may or may not be an associated $P_{i}$, depending on the doubting agent's beliefs supporting $\neg P_{\text {doubt }}$. Lack of a $P_{i}$ indicates that the agent's belief in $\neg P_{\text {doub }}$ is unsupported $^{5}$.

Based on the information that a speaker will ideally convey when expressing doubt (as discussed at the beginning of this section), we lyypothesize that the following aspects of a speaker's beliefs are significant factors in how an expression of doubt is realized.

\subsubsection{Features Associated with $P_{\text {doubt }}$}

Endorsement of $P_{\text {doubt }}$ : Refers to the authority behind the asserted proposition, which impacts the strength of the hearcr's doubt(Chu-Carroll and Carberry, 1998)

- Expert - The information is coming from a domain expert, or coming from someone with firsthand knowledge (including personal preferences).

- Reliable - The agent suggesting the proposition is not an expert, but is considered a generally knowledgeable source.

- Questionable - Information that doesn't come from an expert or reliable agent, or that is stated uncertainly by such an agent.

\subsubsection{Features Associated with $P_{e}$}

$P_{c}$ feature: $P_{e}$ refers to a violated expectation. In the following, we identify three kinds of expectations that may be violated by an assertion. For illustrative purposes, assume that $S$ has made the following assertion:

\section{$\mathrm{S}$ : The most you will get back on your taxes is $\$ 400$.}

- Term-value:

$P_{e}=$ False $\left(. P_{\text {doubt }}\right.$, _term, _value $)$

\footnotetext{
${ }^{5}$ Although human agents may genorally be able to offer some weak support for their beliefs, it is possible, depending on the beliof revision system used, to have to supporting evidence for a belief (Logan et al., 1994).
}

The doubting agent may fail to accept $-P_{\text {doubt }}$ with term instantiated to _value, due to an expectation that svalue is not one of the instantiations of _term that would make $-P_{\text {doubt }}$ true. For cxample, the hearer of the above asscrtion by $\mathrm{S}$ may have expected a much larger value than $\$ 400$, with little or no support for this expectation.

- Constraint:

$P_{c}=$ False (_P doubt, _term, .value, _constraint $)$

The doubting agent may fail to accept.$P_{\text {doub }}$ due to an expectation that ${ }_{-} P_{\text {doubt }}$ will be false when term is instantiated with value, in situations in which _constraint holds. This constraint is not a term in $P_{\text {doubt }}$, but the doubting agent believes that the speaker of $P_{\text {doubt }}$ intends that the constraint hold. For example, the hearer of the above assertion by $\mathrm{S}$ may believe that S means $\$ 400$ for the whole year, but may have expected a larger amount mless $S$ was referring to, say, quarterly taxes.

- Gencral:

$P_{e}=$ False $\left(-P_{\text {doubt }}\right)$

The doubting agent may fail to accept $P_{\text {doubt }}$ in its entirety without having a specific objection to any particular term in the proposition.

\subsubsection{Features Associated with $P_{i}{ }^{6}$}

Commonality of $P_{i}$ refers to the source of the doubting agent,'s conflicting belicf, if any.

- Old - A prior conflicting belief is already part of the explicit common ground of the dialogue.

- New - The doubting agent doesn't believe that; her conflicting belief is alrcady part, of the common ground established by the preceding dialogue.

Endorsement of $P_{i}$ refers to the strength of evidence supporting the belief $P_{i}$ that is in conflict with the doubted belief. The endorsements are listed here from strongest to weakest.

- First-hand - Belief is a porsonal preference or something directly experienced.

- Expert - Bolief supported by expert testimony, or thought to be common knowledge among experts in this domain.

- Roliable - Belief communicated from somconc who, while not an expert, is generally considered a knowledgcable source of information.

- Default - Belief belicved to be common knowledge, in the sense that the speaker strongly believes it and strongly believes that others who belong to a certain community (namely one which she has in common with the other dialogue agent) believe it as woll.

\footnotetext{
${ }^{6}$ We make the simplifying assumption that only one such proposition has been identified for use in an expression of doubt, as this is the case in all of the expressions of doubt we encountered in our corpus. We leave consideration of expressing multiple $P_{i}$ 's in one utterance for future work.
} 
- Derived - Belief is clerived from other bolicfs in such a way that it is considered strong.

- Hypothesis - Tho belief is derived from other bclicfis in such a way that it is considered weak. This category includes belicfs derived from analogy with another belief in a similar proposition.

- Nono - The bolief is unsupported.

Endorsement; of Implication refers to the strength of evidence supporting the belief that $P_{i}$ being true implies that $P_{\text {dout }}$ is not true. 'The ondorsements are listed fiom strongest to weakest. We assume the same definitions as the category above and that the two lists lie on the same strength scale. That is, an implication endorsed as reliable is the same strength as a $P_{i}$ endorsed as reliable and stronger than a $P_{i}$ endorsed as hypothesis. The only addition to this list is the Logical endorsement to acconnt for instances in which $P_{\text {doubt }}$ can be logically defuced from $P_{i}$.

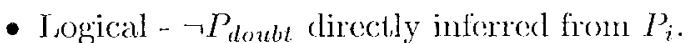

- First-hand 7

- Expcrt

- Roliablo

- Default

- Derived

- Iypothesis

- None.

\subsection{Form ficatures}

Expressions of doubt occur in a variety of forms. Wo distingnish them according to the surface form of the ut terance, the presence of two che words, and the specilicity of the information conveyed.

\section{Surface Form}

- Surface Nogative Question - "Isn't that only wotth what, someone will pay for it?" This category also includes nogative tag questions.

- Simple interrogative - "Can I join the IRA when I am 65 ?"

- Statement as Question - "I must file a return?" 'This category also includes olliptical fragnents such as "\$400?"

- Simple declaration - "I calculated 10."

- Proposition within a bolicf clause - "I thought they only started this yoar."

\section{Clue word}

- But;

- Even though

\footnotetext{
The question of how much experience is needed to learn a belief in an implication is beyond the scope of this paper.
}

Specificity - General forms of the expression can be more or less specific in the amount of information conveyed.

- Generic: Sentence that is a general question of the provious ntterance.

h. you still -you have to file a state income tax return as well

j. i do?

- Repetition: Repetition of a phrase from previous utterance.

h. OK, what I'd like you to do is lend him the 20 thousand.

\section{20 thousand?}

- Repetitiont : Repetition of phrase from previous ntterance plus new information

1. right. the maximum amount of credit that you will be able to get will be $\$ 400$ on their tax return

c. $\$ 400$ for the whole year?

- Contradict: Presentation of a belief that inplies the negation of $P_{\text {doubt }}$

h. and … there's no reason why you shouldn't have an ira for last $y r$

j. well i thought they just started this yr

- Contradict+Sonrce: Presentation of a contradictory belief and the sonrec of that belied

h laad told j he must pay tiax on his $\$ 6256$

j. mm. harry another thing. i have the internal wh revenue wh ask you about that 6256 $\$$ wh since i have the fund he said no! so what do i do now?

- Explicit+Contradict: Fxplicit statement of disbelief, followed by a contradictory belief.

b. well ah he wh ... he belongs to a money market fund now and wh they will do that linvest it in govt securities as part of their individual retirement account for him

h. i'm not so sure of it.. they may move it ah into a into a govt securities fund, but i'm not so sure that they can move it into individual securities - clicck that out

\subsection{Realizing an Expression of Doubt}

Many of the expressions of doubt in ouv corpus are non-ideal, because they were not recognized as doubt or bocause information that was not included in the utterance, but could have been, was altimately needed to resolve the doubt. 'Thus it was not, appropriate to use the corpus as training data for machino learning. Consequently, the following rules are based 
and implication beliefs that would have caused the form of expression of cloubt to be gencrated. We also encouraged subjects to write in beliefs which were not included, but none did. Out of the 60 instances (tien expressions of doubt times six subjects), the subjects five times chose beliefs that we did not represent as contributing to the doubt and three times failed to recognize a belief that did contribute.

The subjects also rated the beliefs according to strength. We evaluated these ratings to sec if the communicated strengths were correlated with the endorsements of beliefs that would have gencrated this form. Since subjects varied in the ranges that they used in rating the strengths of the belicfs, we looked at the scores relative to each subjects ratings of the other beliefs.

Most of the strength ratings were consistent with the rules. 'The most frequent inconsistency was the case in which we would have generated a form based on slightly different endorsements for $P_{i}$ and the implication, but our subjects rated them equivalent. strengths. While it may be the caso that people don't actually perceive a difference, it may also be the case that numerical ratings don't fully capture the same information that the notion of endorsements do.

The only significant inconsistency with our rules was one uttcrance in which doubt was expressed by "I thought, that, but my husband, he wasn't sure if that just meant ss pension." Wo had represented the husband as a reliable source and thus generated information about the source of the conflicting proposition. In this instance, the donbt was not judged very strong by our subjects, and the agent's belief in her husband's oxpertise as relatively weak. In future work, we will further explore expressions of doubt for which it is important to communicate the source of information.

We consider this a preliminary evaluation to show that the rules we have formulated thus far are reasonable. Further evaluation will be neoded to provide evidence that subjects really do draw different inferences based on the clifferent forms of expression and that our rules accurately capture these differences.

\section{Conclusion and Future work}

This paper has presented rules that could be used by a natural language system to realize expressions of doubt. Wo have identified several forms that are used to express doubt in naturally occuring dialogues. Our rules correlate these forms to beliefs of the doubting agent, taking into consideration the beliefs that must be conveyed for the utterance to be a successful expression of doubt.

Proliminary evaluation shows that the belief feature values in our rules correspond to human sub- jects' intuitions about the strength of the doubt. In addition, the beliefs that; would generate cach form are consistent with the beliefs that the subjects at-tribute to the cloubting agent when that form was used.

Future work will concentrate on refining the features and exploring more explicit reasoning about; the beliefs of the other agent. Wo also plan to explore the role of intonation in realizing expressions of doubt.

\section{References}

Jennifer Chu-Carroll and Sanclra Carberry. 1995. Commmication for conflict resolution in multiagent collaborative planning. In Procedings of the International Conference on Multi-Ageni Systems, pages $49-50$.

Jemnifor Chu-Carroll and Sandra Carberry. 1998. Collaborative response generation in planning dialogues. Computational Linguistics, 24(3):355 400.

Herbert Clark. 1996. Using Language. Cambridge University Press.

Julia Rose Galliers. 1992. Autonomous belief revision and communication. In P. Gardenfors, editor; Belief Revision, Cambridge tracts in theoretical computer science. Cambridge University Press, Cambridge, England.

H. Panl Grice. 1975. Logic and Conversation. In P. Cole and J. L. Morgan, clitors, Syntax and Semantics III: Speech Acts, pages 41 58, N.Y. Academic Press.

Lymn Lambert and Sandra Carbory. 1999. A process model for recognizing communicative acts and modeling negotiation subdialogues. Computational Linguistics, 25:1-54.

Brian Logan, Steven Reece, Allison Cawsey, Julia Galliers, and Karen Sparck Jones. 1994. Belief revision and dialogue managenent in information retrieval. Technical report, University of Cambridge Computer Laboralory

IIarry Gross Transcripts. 1982. Transcripts derived from tapes of the radio talk show harry gross: Speaking of your money. Provided by the Dept. of Computer Science at the University of Pennsyl vania.

Keith Vander Linden and Barbara Di Eugenio. 1990. A corpus study of negative imperatives in naturai language instructions. In Procedings of the $10 \mathrm{th}$. International Conference on Computational $J_{\lrcorner} i n$ gusitics (COLING-96), Copenhagen. 